\title{
Retraction Note: Titanium particles damage osteocytes and inhibit osteoblast differentiation
}

\author{
Li Chen ${ }^{1}$, Ziyue Wang ${ }^{2}$, Wei Xu ${ }^{2}$ and Qirong Dong ${ }^{2^{*}}$
}

\section{Correction to: Journal of Experimental Orthopaedics}

7, 47 (2020)

https://doi.org/10.1186/s40634-020-00268-0

The authors have retracted this article [1] because after publication it became apparent that there were problems with the method used for the cultivation of the osteocytes. The data presented are therefore unreliable. All authors agree with this retraction.

\section{Author details}

'Second Department of Orthopaedics, SuZhou Municipal Hospital, Suzhou

City, Anhui Province, China. ${ }^{2}$ Department of Orthopedics, The Second

Affiliated Hospital of Soochow University, Suzhou City, Jiangsu Province,

China.

Published online: 03 November 2020

\section{Reference}

1. Chen L, Wang Z, Xu W et al (2020) Titanium particles damage osteocytes and inhibit osteoblast differentiation. J Exp Ortop 7:47 https://doi.org/10. 1186/s40634-020-00268-0 